Mitsubishi-Tanabe, MSD, Ono, Taisho-Toyama, Takeda, Speakers bureau: Abbvie, Asahi-kasei, Astellas, Bristol-Myers Squibb, Chugai, Daiichi-Sankyo, Eli Lilly, Eisai, Glaxo-Smithkline, Janssen, Mitsubishi-Tanabe, Novartis, Pfizer Japan Inc, Sanofi, Takeda, UCB, YL Biologics DOI: 10.1136/annrheumdis-2019-eular.4562

\section{AB0291E THE EFFECT OF HUMAN UMBILICAL CORD MESENCHYMAL STEM CELLS-DERIVED EXOSOMES ON CHEMOKINES IN COLLAGEN-INDUCED ARTHRITIS RATS}

Ping He. Shanxi Academy of Medical Sciences Shanxi Dayi Hospital, Taiyuan, China

Background: Umbilical cord mesenchymal stem cells (UCMSC) derived exosomes could simulate the function of MSC and avoid the limitations of MSC, which is being hotspot in the research of rheumatoid arthritis (RA) treatment. Chemokines can recruit inflammatory cells and osteoclasts in inflammatory joints, and participate in the synovial inflammation and bone destruction of RA. The mechanisms of UCMSC- derived exosomes on chemokines have less understood in $R A$

Objectives: The aim of this study was to investigate the effect of UCMSC and UCMSC-derived exosomes on the chemokines CCL2, CXCL10 and CXCL12 in CIA rats.

Methods: Human umbilical cord mesenchymal stem cells (UCMSCs) were cultured in vitro and separated using a differential centrifugation methods. The CIA rats model was set up by freund's complete adjuvant and type II collagen, then randomly divided into control, CIA, MTX, UCMSCs, UCMSCs exosomes low and high concentration groups. Rats in UCMSCs group were injected in double ankle joint with UCMSCs $2 \times 10^{6} / \mathrm{L}$ weekly for 3 weeks. Rats in UCMSCs exosomes low and high group were

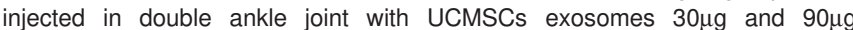
weekly for 3 weeks, respectively. Rats in MTX group were given intraperitoneal injection with MTX $(0.9 \mathrm{mg} / \mathrm{kg})$ weekly for 3 weeks. Serum concentrations of CCL2 and CXCL10 were detected by flow cytometry. CXCL12 serum level was tested by enzyme-linked immunosorbent assay (ELISA). The protein expressions of CCL2, CXCL10 and CXCL12 in synovial tissue were detected by immunohistochemistry. CCL2, CXCL10 and CXCL12 mRNA levels in synovial joint and spleen were measured by reverse transcription-polymerase chain reaction (RT-PCR).

Results: Intra-articular injection of UCMSC exosomes decreased CCL2 and CXCL12 levels in serum and improved synovial hyperplasia and inflammatory cell infiltration in the CIA rats. UCMSC exosomes suppressed the protein expressions of CCL2, CXCL10, CXCL12 in synovial tissue. It also inhibited transcript levels of CCL2, CXCL10, CXCL12 in synovial tissue and spleen. UCMSC had similar effect on CCL2, CXCL10, CXCL12 in CIA. The high concentration group was more effective than the low concentration in preventing CCL2,CXCL10,CXCL12 protein expressions of synovial tissue and CCL2 transcript level of spleen. Conclusion: UCMSC exosomes alleviates synovial inflammation in CIA rats through suppression of CCL2, CXCL10, CXCL12 release. The inhibitory effect of on chemokines simulate UCMSC, their cell of origin, the high concentration was better than low concentration.

\section{REFERENCES}

[1] F. Djouad, V. Fritz, F. Apparailly, P.Reversal of the immunosuppressive properties of mesenchymal stem cells by tumor necrosis factor $\alpha$ in collagen-induced arthritis[J]. Arthritis Rheum., 52 (5) (2005), pp. 1595-1603.

[2] A. Augello, R. Tasso, S.M. Negrini, R. Cell therapy using allogeneic bone marrow mesenchymal stem cells prevents tissue damage in collageninduced arthritis[J]. Arthritis Rheum., 56 (4) (2007), pp. 1175-1186.

[3] Y. Liu, R. Mu, S. Wang, L. Long, Therapeutic potential of human umbilical cord mesenchymal stem cells in the treatment of rheumatoid arthritis[J]. Arthritis Res Ther., 12 (6) (2010), p. R210.

[4] Rani S,Ryan AE,Griffin MD, Ritter T.Mesenchymal stem cell-derived extracellular vesicles:toward cell-free therapeutic applications[J].Mol Ther,2015,23(5):8 12-823

[5] Szekanecz Z, Kim J, Koch AE. Chemokines and chemokine receptors in rheumatoid arthritis. Semin Immunol. 2003 Feb;15(1):15-21.

Disclosure of Interests: None declared

DOI: 10.1136/annrheumdis-2019-eular.7165

\section{Rheumatoid arthritis - comorbidity and clinical aspects}

\section{AB0292 EFFECTS OF ACETAMINOPHEN ON THE KIDNEY FUNCTIONS OF PAITIENTS WITH MUSCULOSKERLTAL DISEASE TREATED WITH LONG-TERM NONSTEROIDAL ANTI-INFRAMMATORY DRUG THERAPY}

Toshiaki Aizawa $^{1}$, Tetsuya Kawano ${ }^{1}$, Takeshi Kashiwagura ${ }^{2}$, Naohisa Miyakoshi ${ }^{3}$, Yoichi Shimada ${ }^{3} .{ }^{1}$ Kitaakita Municipal Hospital, Akita, Japan; ${ }^{2}$ Akita City Hospital, Akita, Japan; ${ }^{3}$ Akita University Graduate School of Medicine, Akita, Japan

Background: While nonsteroidal anti-inflammatory drugs (NSAIDs) are the most frequently used therapeutic agents, they are known to cause side effects, such as gastrointestinal disorders and kidney dysfunction. As the proportion of patients with chronic kidney disease (CKD) increases with age, dealing with NSAIDs requires particular care in areas with aging populations. However, in many cases, prescriptions are continued without any particular plan. Hence, the effects of acetaminophen (AAP), which does not cause kidney dysfunction, are being revisited. Opportunities are increasing for using formulations containing tramadol hydrochloride in patients with chronic pain or intravenous agents for perioperative pain management.

Objectives: In this study, we investigated the kidney functions of patients with musculoskeletal disease who had been using NSAIDs for a long time and examined the possibility of switching to AAP.

Methods: The subjects were 105 patients with musculoskeletal disease (42 men and 63 women) who were being treated as outpatients in the orthopedics department of our hospital and had been using NSAIDs for at least 3 months. They primarily had 5 musculoskeletal diseases, including degenerative osteoarthritis (OA), rheumatoid arthritis (RA), osteoporosis $(\mathrm{OP})$, polymyalgia rheumatica (PMR), and gout $(\mathrm{G})$. After checking their background characteristics, we measured their kidney functions and visual analogue scale (VAS) scores, and then switched them from NSAIDs to AAP. Kidney functions were evaluated by classifying CKD using glomerular filtration rate (eGFR) and urinary protein, and by using the CockcroftGault equation to calculate creatinine clearance (Ccr). The patients were given the NSAIDs that they had been taking for single use. Six months after the switch, we remeasured their kidney functions and VAS scores, and investigated whether they could continue using AAP and whether they had used NSAIDs.

Results: The patients' mean age at the time of switching from NSAIDs to AAP was 76 years, and their mean duration of NSAID use was 43 months. For the diseases, 63 patients had OA, 20 had RA, 14 had OP 6 had PMR, and 2 had G. Before the switch, the mean VAS score was $41.6 \mathrm{~mm}$ and mean eGFR was $67.0 \mathrm{~mL} / \mathrm{min} / 1.73 \mathrm{~m}^{2}$. On the basis of the eGFR, the CKD classification was G3 or higher in $38.1 \%$ of patients. The CKD classifications with the amounts of urinary protein added to the eGFR were as follows: green, $44.7 \%$; yellow, $33.0 \%$; orange, $14.6 \%$; and red, $7.8 \%$, with $55.4 \%$ classified as having CKD. By age, the proportions of patients with CKD based on eGFR and CKD classification were as follows, respectively: $0.0 \%$ and $12.5 \%$ for those in their $50 \mathrm{~s}, 16.7 \%$ and $25.0 \%$ for those in their $60 \mathrm{~s}, 29.3 \%$ and $48.8 \%$ for those in their $70 \mathrm{~s}$ and $59.5 \%$ and $69.0 \%$ for those in their $80 \mathrm{~s}$ and older. The mean AAP dose was $455 \mathrm{mg}$.

Six months after the switch, 69 patients had continuously used AAP without regular NSAID use. Of these patients, 13 had used NSAIDs for rescue purposes. Thirty-six patients had stopped using AAP, the most common reason being ineffectiveness (15 patients), although 9 patients did so because they no longer needed any analgesic. Three patients stopped using the drug owing to palpitations or other side effects, although none stopped because of liver dysfunction. Overall, the mean VAS score declined significantly as compared with before the switch, as did the mean eGFR. Of those who continued to use AAP, 56 patients who did not use any NSAIDs exhibited significantly lower mean VAS scores, but not a significant decline in mean eGFR.

Conclusion: The proportion of patients with CKD who were receiving long-term NSAID therapy was higher than that of the general population for all age groups. Halting NSAIDs and switching to AAP could help maintain kidney functions, which could continue to decline with NSAID use.

\section{REFERENCES}

[1] CKD guidebook;2012 